\title{
INVESTIGATION OF STRESS-STRAIN STATE OF TRANSVERSELY ISOTROPIC PLATES UNDER BENDING USING EQUATION OF STATICS $\{1,2\}$-APPROXIMATION
}

\author{
Igor Bokov \\ Postgraduate student \\ Department of Strength and Optimization \\ A. N. Podgorny Institute of Mechanical Engineering Problems NAS of Ukraine \\ 2/10 Pozharsky str., Kharkiv, Ukraine, 61046 \\ igp.bokov@gmail.com \\ Natalia Bondarenko \\ PhD \\ Bondarenko.Natalya.Sergeevna@gmail.com \\ Elena Strelnikova \\ Doctor of Technical Sciences, professor \\ Department of Strength and Optimization \\ A. N. Podgorny Institute of Mechanical Engineering Problems NAS of Ukraine \\ 2/10 Pozharsky str., Kharkiv, Ukraine, 61046 \\ elena15@gmx.com
}

\begin{abstract}
The study examined the construction of the fundamental solution for the equations of statics $\{1,2\}$-approximation for transversely isotropic plates under bending with the action of concentrated force. Equations $\{1,2\}$-approximation were obtained by the decomposition method in the thickness coordinate using the Legendre polynomials. These equations take into account all the components of the stress tensor, including the transverse shear and normal stresses. Since the classical theory of Kirchhoff-Love doesn't take account of these stresses, the study on the basis of refined theories of stress-strain state of transversely isotropic plates under the action of concentrated force effects is an important scientific and technical problem.

The fundamental solution of obtained equations results using a two-dimensional Fourier integral transform and inverse treatment techniques, built with the help of a special G-function. This method allows reducing the system of resolving differential equations for statics of flat plates and shells to a system of algebraic equations. After that, the inverse Fourier transform restores the fundamental solution. The work was carried out numerical studies that demonstrate patterns of behavior of components of the stress-strain state, depending on the elastic constants of transversely isotropic material. The results play a decisive role in the study of boundary value problems in the mechanics of thin-walled elements of constructions, including under the influence of concentrated and local diverse forces.
\end{abstract}

Keywords: $\{1,2\}$-approximation, equations of statics, transversely isotropic plates, concentrated force, bending state.

\section{Introduction}

In modern technology, engineering structures with thin-walled structural elements are widely used. There are design of aircraft, such as a solid-fuel rocket engine (SFRE), and liquid-fuel rocket engine (LFRE) [1] under considerable force effects. In modern engineering new composite materials are intensively used for creating protective coatings on the friction surfaces and for the manufacture of various items of equipment [2]. The use of such materials makes it necessary to build a refined theory of plates and shells, taking into account the phenomena associated with the transverse shear and compression.

To reducing the three-dimensional problem for transversely isotropic plates to the twodimensional problem, generalized theory of $\{\mathrm{m}, \mathrm{n}\}$-approximation is used in the article. The selected method is the most appropriate for this task, because it is not based on any hypotheses, and uses the method of Vekua decomposition of unknown functions in Fourier series using Legendre polynomials [3]. This approach allows considering not only the thin plate, but the plate of medium 
and high thickness. The accuracy of these solutions depends on the number of retained items in the expansions of the given and unknown functions.

Generalized theory of plates and shells in the variant of $\{1,2\}$-approximation is used in the article for derivation of static equations for transversely isotropic plates under bending with action of concentrated force. In this paper, a fundamental solution to the equations of statics $\{1,2\}$-approximation is obtained.

This problem must be solved as fundamental solutions play a decisive role in the study of boundary problems in the mechanics of thin-walled elements of constructions, including under the influence of a variety of local and concentrated forces such as local force impact.

Among recent publications that use the generalized theory of $\{\mathrm{m}, \mathrm{n}\}$-approximation, it should be noted the articles on problems of statics $[4,5]$, as well as publications that addressed the problem of thermoelasticity [6-9].

Bending problem for transversely isotropic plates using the equations of statics $\{1,2\}$ approximation is solved below.

\section{Materials and methods}

Approximation method of the displacement, stress and strain of the Fourier series using Legendre polynomials on the transverse coordinate to derivation of two-dimensional equations of statics for transversely isotropic plates is used. This method is the most preferred, as it allows obtaining two-dimensional equations of statics is not based on any hypotheses, and by expanding the unknown functions. The fundamental solution of the obtained equations of statics $\{1,2\}$-approximation found with the help of a two-dimensional Fourier integral transform.

\section{Experimental investigations}

3. 1. Basic relations and mathematical formulation of the static problem, describing the state of bending transversely isotropic plates based on $\{1,2\}$-approximation

Let's consider a transversely isotropic plate with $2 \mathrm{~h}$ thickness in a rectangular Cartesian coordinate system $\mathrm{x}, \mathrm{y}, \mathrm{z}$. Concentrated force $\overrightarrow{\mathrm{F}}$ applied at the origin (singular point) acts on the plate.

As part of $\{1,2\}$-approximation there are representations for components of the displacement vector and the stress tensor under bending [3]

$$
\begin{gathered}
\mathrm{u}_{\mathrm{x}}=\gamma_{\mathrm{x}} \mathrm{hP}_{1}, \mathrm{u}_{\mathrm{y}}=\gamma_{\mathrm{y}} \mathrm{hP}_{1}, \mathrm{u}_{\mathrm{z}}=\mathrm{w}_{0} \mathrm{P}_{0}+\mathrm{w}_{2} \mathrm{P}_{2}, \\
\sigma_{\mathrm{x}}=\frac{3 \mathrm{M}_{\mathrm{x}}}{2 \mathrm{~h}^{2}} \mathrm{P}_{1}(\mathrm{x} \rightarrow \mathrm{y}), \tau_{\mathrm{xy}}=\frac{3 \mathrm{H}}{2 \mathrm{~h}^{2}} \mathrm{P}_{1}, \\
\tau_{\mathrm{xz}}=\frac{\mathrm{Q}_{\mathrm{x} 0}}{2 \mathrm{~h}}\left(\mathrm{P}_{0}-\mathrm{P}_{4}\right)+\frac{5 \mathrm{Q}_{\mathrm{x} 2}}{2 \mathrm{~h}}\left(\mathrm{P}_{2}-\mathrm{P}_{4}\right)(\mathrm{x} \rightarrow \mathrm{y}), \sigma_{\mathrm{z}}=\frac{3 \mathrm{R}_{1}}{2 \mathrm{~h}}\left(\mathrm{P}_{1}-\mathrm{P}_{3}\right), \\
\mathrm{F}_{\mathrm{x}}=\frac{3 \mathrm{~m}_{\mathrm{x}}}{2 \mathrm{~h}^{2}} \mathrm{P}_{1}(\mathrm{x} \rightarrow \mathrm{y}), \mathrm{F}_{\mathrm{z}}=\frac{\mathrm{q}_{\mathrm{z} 0}}{2 \mathrm{~h}} \mathrm{P}_{0}+\frac{5 \mathrm{q}_{\mathrm{z} 2}}{2 \mathrm{~h}} \mathrm{P}_{2},
\end{gathered}
$$

where $\mathrm{w}_{\mathrm{j}}(\mathrm{j}=0,2), \gamma_{\mathrm{i}}(\mathrm{i}=\mathrm{x}, \mathrm{y})$ - generalized displacement of the plate; $\gamma_{\mathrm{i}}(\mathrm{i}=\mathrm{x}, \mathrm{y})$ are analogous to the normal rotation angles; $M_{i}(i=x, y), H, Q_{i j}(i=x, y ; j=0,2), R_{1}$ - generalized moments and forces; $\overrightarrow{\mathrm{F}}=\left(\mathrm{F}_{\mathrm{x}}, \mathrm{F}_{\mathrm{y}}, \mathrm{F}_{\mathrm{z}}\right)$ - vector of volume force; $\mathrm{m}_{\mathrm{x}}=\mathrm{m}_{1} \mathrm{Eh}, \mathrm{m}_{\mathrm{y}}=\mathrm{m}_{2} \mathrm{Eh}, \mathrm{q}_{\mathrm{z} 0}=\mathrm{q}_{4} \mathrm{E}$, $\mathrm{q}_{\mathrm{z} 2}=\mathrm{q}_{5} \mathrm{E}$ - decomposition components of the vector of volume force. Among them, $\mathrm{M}_{\mathrm{x}}, \mathrm{M}_{\mathrm{y}}$ are analogues of bending and torque moments; $\mathrm{M}_{\mathrm{x}}, \mathrm{M}_{\mathrm{y}}$ - Legendre polynomials.

The equations of statics in the form $\{1,2\}$-approximation for transversely isotropic plates, describing the bending state, written in the dimensionless coordinate system $\left(\mathrm{x}_{1}=\mathrm{x} / \mathrm{h}, \mathrm{x}_{2}=\mathrm{y} / \mathrm{h}\right.$, $\mathrm{x}_{3}=\mathrm{z} / \mathrm{h}$ ) and contain [3]:

- Equations of Hooke's law:

$$
\mathrm{M}_{1}=\mathrm{D}_{0}\left(\frac{\partial \gamma_{1}}{\partial \mathrm{x}_{1}}+v \frac{\partial \gamma_{2}}{\partial \mathrm{x}_{2}}\right)+\lambda_{0} \mathrm{R}_{1}, \mathrm{M}_{2}=\mathrm{D}_{0}\left(\frac{\partial \gamma_{2}}{\partial \mathrm{x}_{2}}+v \frac{\partial \gamma_{1}}{\partial \mathrm{x}_{1}}\right)+\lambda_{0} \mathrm{R}_{1}
$$




$$
\begin{gathered}
\mathrm{H}=\frac{1-\mathrm{v}}{2} \mathrm{D}_{0}\left(\frac{\partial \gamma_{2}}{\partial \mathrm{x}_{1}}+\frac{\partial \gamma_{1}}{\partial \mathrm{x}_{2}}\right) \\
\mathrm{Q}_{10}=\Lambda_{0}\left[\gamma_{1}+\frac{\partial}{\partial \mathrm{x}_{1}}\left(\mathrm{w}_{0}-\frac{\mathrm{w}_{2}}{14}\right)\right], \mathrm{Q}_{20}=\Lambda_{0}\left[\gamma_{2}+\frac{\partial}{\partial \mathrm{x}_{2}}\left(\mathrm{w}_{0}-\frac{\mathrm{w}_{2}}{14}\right)\right] \\
\mathrm{Q}_{12}=-\frac{\Lambda_{0}}{14}\left[\gamma_{1}+\frac{\partial}{\partial \mathrm{x}_{1}}\left(\mathrm{w}_{0}-2 \mathrm{w}_{2}\right)\right], \mathrm{Q}_{22}=-\frac{\Lambda_{0}}{14}\left[\gamma_{2}+\frac{\partial}{\partial \mathrm{x}_{2}}\left(\mathrm{w}_{0}-2 \mathrm{w}_{2}\right)\right], \\
\mathrm{R}_{1}=\Omega_{0}\left[\mathrm{w}_{2}+\frac{\lambda}{3}\left(\frac{\partial \gamma_{1}}{\partial \mathrm{x}_{1}}+\frac{\partial \gamma_{2}}{\partial \mathrm{x}_{2}}\right)\right]
\end{gathered}
$$

where

$\mathrm{D}_{0}=\frac{2}{3} \frac{1}{1-v^{2}}, \lambda_{0}=\frac{v^{\prime}}{1-v} \mathrm{E}^{*}, \Lambda_{0}=\frac{28}{15} \frac{1}{\mathrm{E} / \mathrm{G}^{\prime}}, \Omega_{0}=\frac{7}{5} \frac{(1-\mathrm{v}) / \mathrm{E}^{*}}{1-\mathrm{v}-2\left(\mathrm{v}^{\prime}\right)^{2} \mathrm{E}^{*}}, \mathrm{E}^{*}=\frac{\mathrm{E}}{\mathrm{E}^{\prime}}$.

$\mathrm{E}, \mathrm{E}^{\prime}$ - Young's modulus for directions in the plane of isotropy and perpendicular to it; $v, v^{\prime}, G, G^{\prime}$ - Poisson's ratio and shear modulus corresponding to these directions;

- Equilibrium equations

$$
\begin{gathered}
\frac{\partial \mathrm{M}_{1}}{\partial \mathrm{x}_{1}}+\frac{\partial \mathrm{H}}{\partial \mathrm{x}_{2}}-\mathrm{Q}_{10}+\mathrm{m}_{1}=0, \frac{\partial \mathrm{M}_{2}}{\partial \mathrm{x}_{2}}+\frac{\partial \mathrm{H}}{\partial \mathrm{x}_{1}}-\mathrm{Q}_{20}+\mathrm{m}_{2}=0 \\
\frac{\partial \mathrm{Q}_{10}}{\partial \mathrm{x}_{1}}+\frac{\partial \mathrm{Q}_{20}}{\partial \mathrm{x}_{2}}+\mathrm{q}_{4}=0, \frac{\partial \mathrm{Q}_{12}}{\partial \mathrm{x}_{1}}+\frac{\partial \mathrm{Q}_{22}}{\partial \mathrm{x}_{2}}-3 \mathrm{R}_{1}+\mathrm{q}_{5}=0
\end{gathered}
$$

where $\mathrm{m}_{1}=\mathrm{m}_{1}^{*} \delta\left(\mathrm{x}_{1}, \mathrm{x}_{2}\right), \mathrm{m}_{2}=\mathrm{m}_{2}^{*} \delta\left(\mathrm{x}_{1}, \mathrm{x}_{2}\right), \mathrm{q}_{4}=\mathrm{q}_{4}^{*} \delta\left(\mathrm{x}_{1}, \mathrm{x}_{2}\right), \mathrm{q}_{5}=\mathrm{q}_{5}^{*} \delta\left(\mathrm{x}_{1}, \mathrm{x}_{2}\right), \delta\left(\mathrm{x}_{1}, \mathrm{x}_{2}\right)-$ twodimensional Dirac delta function [10].

Moments in (1) are defined up to a value of $\mathrm{Eh}^{2}$, and generalized forces - up to Eh.

\section{2. Construction of the fundamental solution for equations of statics in the $\{1,2\}$-ap-} proximation

Substituting the equations of Hooke's law (1) in the equilibrium equations (2) and applying the Fourier transform to the resulting system, we obtain a system of linear algebraic equations in the space of transformants $\left(\xi_{1}, \xi_{2}\right)$

$$
\begin{gathered}
\left(\mathrm{A}_{1} \xi_{1}^{2}+\frac{1-\mathrm{v}}{2} \mathrm{D}_{0} \xi_{2}^{2}+\Lambda_{0}\right) \tilde{\gamma}_{1}+\mathrm{A}_{2} \xi_{1} \xi_{2} \tilde{\gamma}_{2}-\Lambda_{0} \mathrm{i} \xi_{1} \tilde{\mathrm{w}}_{0}+\mathrm{A}_{3} \mathrm{i} \xi_{1} \tilde{\mathrm{w}}_{2}=\frac{\mathrm{m}_{1}^{*}}{2 \pi} \\
\mathrm{A}_{2} \xi_{1} \xi_{2} \tilde{\gamma}_{1}+\left(\frac{1-\mathrm{v}}{2} \mathrm{D}_{0} \xi_{1}^{2}+\mathrm{A}_{1} \xi_{2}^{2}+\Lambda_{0}\right) \tilde{\gamma}_{2}-\Lambda_{0} \mathrm{i} \xi_{2} \tilde{\mathrm{w}}_{0}+\mathrm{A}_{3} \mathrm{i} \xi_{2} \tilde{\mathrm{w}}_{2}=\frac{\mathrm{m}_{2}^{*}}{2 \pi}, \\
\Lambda_{0} \mathrm{i}\left(\xi_{1} \tilde{\gamma}_{1}+\xi_{2} \tilde{\gamma}_{2}\right)+\Lambda_{0} \mathrm{p}^{2} \tilde{\mathrm{w}}_{0}-\frac{1}{14} \Lambda_{0} \mathrm{p}^{2} \tilde{\mathrm{w}}_{2}=\frac{\mathrm{q}_{4}^{*}}{2 \pi}, \\
-\mathrm{A}_{3} \mathrm{i}\left(\xi_{1} \tilde{\gamma}_{1}+\xi_{2} \tilde{\gamma}_{2}\right)-\frac{1}{14} \Lambda_{0} \mathrm{p}^{2} \tilde{\mathrm{w}}_{0}+\left(3 \Omega_{0}+\frac{1}{7} \Lambda_{0} \mathrm{p}^{2}\right) \tilde{\mathrm{w}}_{2}=\frac{\mathrm{q}_{5}^{*}}{2 \pi},
\end{gathered}
$$

where

$$
\mathrm{A}_{1}=\mathrm{D}_{0}+\frac{1}{3} \lambda_{0}^{2} \Omega_{0}, \mathrm{~A}_{2}=\mathrm{A}_{1}+\frac{v-1}{2} \mathrm{D}_{0}, \mathrm{~A}_{3}=\lambda_{0} \Omega_{0}+\frac{1}{14} \Lambda_{0}
$$

Solving this system, we can find generalized displacements in the space of transformants: 


$$
\begin{aligned}
& \tilde{\gamma}_{1}=\frac{1}{2 \pi \mathrm{D}_{0}}\left\{\mathrm{~m}_{1}^{*} \mathrm{a}_{1}^{2} \frac{\xi_{1}^{2}}{\mathrm{p}^{4}\left(\mathrm{p}^{2}+\mathrm{a}_{1}^{2}\right)}+\mathrm{m}_{2}^{*} \mathrm{a}_{1}^{2} \frac{\xi_{1} \xi_{2}}{\mathrm{p}^{4}\left(\mathrm{p}^{2}+\mathrm{a}_{1}^{2}\right)}-\right. \\
& -\mathrm{q}_{5}^{*} \frac{\lambda_{0} \mathrm{a}_{1}^{2}}{3} \frac{\mathrm{i} \xi_{1}}{\mathrm{p}^{2}\left(\mathrm{p}^{2}+\mathrm{a}_{1}^{2}\right)}+\mathrm{q}_{4}^{*}\left[\frac{\mathrm{D}_{0}}{\mathrm{~A}_{1}}-\frac{\lambda_{0} \mathrm{a}_{1}^{2}}{42}\right] \frac{\mathrm{i} \xi_{1}}{\mathrm{p}^{2}\left(\mathrm{p}^{2}+\mathrm{a}_{1}^{2}\right)}+ \\
& +\mathrm{m}_{1}^{*} \frac{\mathrm{D}_{0}}{\mathrm{~A}_{1}} \frac{\xi_{1}^{2}}{\mathrm{p}^{2}\left(\mathrm{p}^{2}+\mathrm{a}_{1}^{2}\right)}+\mathrm{m}_{2}^{*} \frac{\mathrm{D}_{0}}{\mathrm{~A}_{1}} \frac{\xi_{1} \xi_{2}}{\mathrm{p}^{2}\left(\mathrm{p}^{2}+\mathrm{a}_{1}^{2}\right)}+ \\
& \left.+\mathrm{q}_{4}^{*} \mathrm{a}_{1}^{2} \frac{\mathrm{i} \xi_{1}}{\mathrm{p}^{4}\left(\mathrm{p}^{2}+\mathrm{a}_{1}^{2}\right)}-\mathrm{m}_{1}^{*} \frac{2}{v-1} \frac{\xi_{2}^{2}}{\mathrm{p}^{2}\left(\mathrm{p}^{2}+\mathrm{b}_{1}^{2}\right)}+\mathrm{m}_{2}^{*} \frac{2}{v-1} \frac{\xi_{1} \xi_{2}}{\mathrm{p}^{2}\left(\mathrm{p}^{2}+\mathrm{b}_{1}^{2}\right)}\right\}, \\
& \tilde{\gamma}_{2}=\frac{1}{2 \pi D_{0}}\left\{\mathrm{~m}_{1}^{*} \mathrm{a}_{1}^{2} \frac{\xi_{1} \xi_{2}}{\mathrm{p}^{4}\left(\mathrm{p}^{2}+\mathrm{a}_{1}^{2}\right)}+\mathrm{m}_{2}^{*} \mathrm{a}_{1}^{2} \frac{\xi_{2}^{2}}{\mathrm{p}^{4}\left(\mathrm{p}^{2}+\mathrm{a}_{1}^{2}\right)}-\mathrm{q}_{5}^{*} \frac{\lambda_{0} \mathrm{a}_{1}^{2}}{3} \frac{\mathrm{i} \xi_{2}}{\mathrm{p}^{2}\left(\mathrm{p}^{2}+\mathrm{a}_{1}^{2}\right)}+\right. \\
& +\mathrm{q}_{4}^{*}\left[\frac{\mathrm{D}_{0}}{\mathrm{~A}_{1}}-\frac{\lambda_{0} \mathrm{a}_{1}^{2}}{42}\right] \frac{\mathrm{i} \xi_{2}}{\mathrm{p}^{2}\left(\mathrm{p}^{2}+\mathrm{a}_{1}^{2}\right)}+\mathrm{m}_{1}^{*} \frac{\mathrm{D}_{0}}{\mathrm{~A}_{1}} \frac{\xi_{1} \xi_{2}}{\mathrm{p}^{2}\left(\mathrm{p}^{2}+\mathrm{a}_{1}^{2}\right)}+\mathrm{m}_{2}^{*} \frac{\mathrm{D}_{0}}{\mathrm{~A}_{1}} \frac{\xi_{2}^{2}}{\mathrm{p}^{2}\left(\mathrm{p}^{2}+\mathrm{a}_{1}^{2}\right)}+ \\
& \left.+\mathrm{q}_{4}^{*} \mathrm{a}_{1}^{2} \frac{\mathrm{i} \xi_{2}}{\mathrm{p}^{4}\left(\mathrm{p}^{2}+\mathrm{a}_{1}^{2}\right)}+\mathrm{m}_{1}^{*} \frac{2}{\mathrm{v}-1} \frac{\xi_{1} \xi_{2}}{\mathrm{p}^{2}\left(\mathrm{p}^{2}+\mathrm{b}_{1}^{2}\right)}-\mathrm{m}_{2}^{*} \frac{2}{\mathrm{v}-1} \frac{\xi_{1}^{2}}{\mathrm{p}^{2}\left(\mathrm{p}^{2}+\mathrm{b}_{1}^{2}\right)}\right\} \\
& \tilde{\mathrm{w}}_{0}=\frac{1}{2 \pi \mathrm{D}_{0}}\left\{\mathrm{q}_{4}^{*} \frac{28 \mathrm{D}_{0}}{27 \Lambda_{0}} \frac{1}{\mathrm{p}^{2}+\mathrm{a}_{1}^{2}}+\mathrm{q}_{5}^{*} \frac{14 \mathrm{D}_{0}}{27 \Lambda_{0}} \frac{1}{\mathrm{p}^{2}+\mathrm{a}_{1}^{2}}+\mathrm{q}_{4}^{*}\left[\frac{\mathrm{D}_{0} \mathrm{a}_{1}^{2}}{\Lambda_{0}}-\frac{\lambda_{0} \mathrm{a}_{1}^{2}}{21}+\frac{\mathrm{D}_{0}}{\mathrm{~A}_{1}}\right] \times\right. \\
& \times \frac{1}{\mathrm{p}^{2}\left(\mathrm{p}^{2}+\mathrm{a}_{1}^{2}\right)}-\mathrm{q}_{5}^{*} \frac{\lambda_{0} \mathrm{a}_{1}^{2}}{3} \frac{1}{\mathrm{p}^{2}\left(\mathrm{p}^{2}+\mathrm{a}_{1}^{2}\right)}+\mathrm{q}_{4}^{*} \mathrm{a}_{1}^{2} \frac{1}{\mathrm{p}^{4}\left(\mathrm{p}^{2}+\mathrm{a}_{1}^{2}\right)}- \\
& -m_{1}^{*} a_{1}^{2} \frac{i \xi_{1}}{p^{4}\left(p^{2}+a_{1}^{2}\right)}-m_{2}^{*} a_{1}^{2} \frac{i \xi_{2}}{p^{4}\left(p^{2}+a_{1}^{2}\right)}+m_{1}^{*}\left[\frac{\lambda_{0} a_{1}^{2}}{42}-\frac{D_{0}}{A_{1}}\right] \times \\
& \left.\times \frac{i \xi_{1}}{p^{2}\left(p^{2}+a_{1}^{2}\right)}+m_{2}^{*}\left[\frac{\lambda_{0} a_{1}^{2}}{42}-\frac{D_{0}}{A_{1}}\right] \frac{i \xi_{2}}{p^{2}\left(p^{2}+a_{1}^{2}\right)}\right\} \\
& \tilde{\mathrm{w}}_{2}=\frac{1}{6 \pi \mathrm{D}_{0}}\left\{\mathrm{q}_{4}^{*} \frac{14 \mathrm{D}_{0}}{9 \Lambda_{0}} \frac{1}{\mathrm{p}^{2}+\mathrm{a}_{1}^{2}}-\mathrm{q}_{4}^{*} \lambda_{0} \mathrm{a}_{1}^{2} \frac{1}{\mathrm{p}^{2}\left(\mathrm{p}^{2}+\mathrm{a}_{1}^{2}\right)}+\mathrm{q}_{5}^{*} \frac{196 \mathrm{D}_{0}}{9 \Lambda_{0}} \frac{1}{\mathrm{p}^{2}+\mathrm{a}_{1}^{2}}+\right. \\
& \left.+\mathrm{m}_{1}^{*} \lambda_{0} \mathrm{a}_{1}^{2} \frac{\mathrm{i} \xi_{1}}{\mathrm{p}^{2}\left(\mathrm{p}^{2}+\mathrm{a}_{1}^{2}\right)}+\mathrm{m}_{2}^{*} \lambda_{0} \mathrm{a}_{1}^{2} \frac{\mathrm{i} \xi_{2}}{\mathrm{p}^{2}\left(\mathrm{p}^{2}+\mathrm{a}_{1}^{2}\right)}\right\},
\end{aligned}
$$

where $\mathrm{a}_{1}^{2}=\frac{196 \mathrm{D}_{0} \Omega_{0}}{9 \mathrm{~A}_{1} \Lambda_{0}}, \mathrm{~b}_{1}^{2}=\frac{2 \Lambda_{0}}{\mathrm{D}_{0}(1-v)}, \mathrm{p}^{2}=\xi_{1}^{2}+\xi_{2}^{2} ;\left(\xi_{1}, \xi_{2}\right)-$ coordinates of the point in the space of transformants.

Applying the Fourier transform to equations (1) and substituting transformants of generalized displacements (4) in these relations, we can find the expression for the generalized moments and forces. Then, for these expressions in the space of transformants using the inversion formula for the two-dimensional Fourier integral transform [11], we get the originals of internal force factors 


$$
\begin{aligned}
& \mathrm{M}_{1}=-\frac{1}{2 \pi}\left\{\mathrm{m}_{1}^{*} \mathrm{a}_{1}^{2} \Phi_{1}\left(\mathrm{x}_{1}, \mathrm{x}_{2}\right)+\mathrm{m}_{2}^{*} \mathrm{a}_{1}^{2} \Phi_{2}\left(\mathrm{x}_{1}, \mathrm{x}_{2}\right)+\mathrm{q}_{5}^{*} \frac{\lambda_{0} \mathrm{a}_{1}^{2}}{3} \Phi_{3}\left(\mathrm{x}_{1}, \mathrm{x}_{2}, \mathrm{a}_{1}\right)-\right. \\
& -\mathrm{q}_{4}^{*}\left[\frac{\mathrm{D}_{0}}{\mathrm{~A}_{1}}-\frac{\lambda_{0} \mathrm{a}_{1}^{2}}{42}\right] \Phi_{3}\left(\mathrm{x}_{1}, \mathrm{x}_{2}, \mathrm{a}_{1}\right)+\mathrm{m}_{1}^{*} \frac{\mathrm{D}_{0}}{\mathrm{~A}_{1}} \Phi_{4}\left(\mathrm{x}_{1}, \mathrm{x}_{2}, \mathrm{a}_{1}\right)+\mathrm{m}_{2}^{*} \frac{\mathrm{D}_{0}}{\mathrm{~A}_{1}} \Phi_{5}\left(\mathrm{x}_{1}, \mathrm{x}_{2}, \mathrm{a}_{1}\right)- \\
& -\mathrm{q}_{4}^{*} \mathrm{a}_{1}^{2} \Phi_{6}\left(\mathrm{x}_{1}, \mathrm{x}_{2}\right)+2 \mathrm{~m}_{1}^{*} \Phi_{5}\left(\mathrm{x}_{2}, \mathrm{x}_{1}, \mathrm{~b}_{1}\right)-2 \mathrm{~m}_{2}^{*} \Phi_{5}\left(\mathrm{x}_{1}, \mathrm{x}_{2}, \mathrm{~b}_{1}\right)+ \\
& +\mathrm{m}_{1}^{*} \mathrm{a}_{1}^{2} v \Phi_{2}\left(\mathrm{x}_{2}, \mathrm{x}_{1}\right)+\mathrm{m}_{2}^{*} \mathrm{a}_{1}^{2} v \Phi_{1}\left(\mathrm{x}_{2}, \mathrm{x}_{1}\right)+\mathrm{q}_{5}^{*} \frac{\lambda_{0} \mathrm{a}_{1}^{2} \mathrm{v}}{3} \Phi_{3}\left(\mathrm{x}_{2}, \mathrm{x}_{1}, \mathrm{a}_{1}\right)- \\
& -\mathrm{q}_{4}^{*} v\left[\frac{\mathrm{D}_{0}}{\mathrm{~A}_{1}}-\frac{\lambda_{0} \mathrm{a}_{1}^{2}}{42}\right] \Phi_{3}\left(\mathrm{x}_{2}, \mathrm{x}_{1}, \mathrm{a}_{1}\right)+\mathrm{m}_{1}^{*} \frac{v \mathrm{D}_{0}}{\mathrm{~A}_{1}} \Phi_{5}\left(\mathrm{x}_{2}, \mathrm{x}_{1}, \mathrm{a}_{1}\right)+\mathrm{m}_{2}^{*} \frac{v \mathrm{D}_{0}}{\mathrm{~A}_{1}} \Phi_{4}\left(\mathrm{x}_{2}, \mathrm{x}_{1}, \mathrm{a}_{1}\right)- \\
& \left.-\mathrm{q}_{4}^{*} \mathrm{a}_{1}^{2} v \Phi_{6}\left(\mathrm{x}_{2}, \mathrm{x}_{1}\right)\right\}+\frac{\lambda_{0}^{2} \Omega_{0}}{6 \pi \mathrm{A}_{1}}\left\{\mathrm{q}_{4}^{*}\left[1+\frac{\mathrm{A}_{1} \mathrm{a}_{1}^{2}}{14 \lambda_{0} \Omega_{0}}\right] \Phi_{7}\left(\mathrm{x}_{1}, \mathrm{x}_{2}\right)+\mathrm{q}_{5}^{*} \frac{\mathrm{A}_{1} \mathrm{a}_{1}^{2}}{\lambda_{0} \Omega_{0}} \Phi_{7}\left(\mathrm{x}_{1}, \mathrm{x}_{2}\right)-\right. \\
& \left.-\mathrm{m}_{1}^{*} \Phi_{8}\left(\mathrm{x}_{1}, \mathrm{x}_{2}\right)-\mathrm{m}_{2}^{*} \Phi_{8}\left(\mathrm{x}_{2}, \mathrm{x}_{1}\right)\right\} \text {, } \\
& \mathrm{M}_{2}=-\frac{1}{2 \pi}\left\{\mathrm{m}_{1}^{*} \mathrm{a}_{1}^{2} \Phi_{2}\left(\mathrm{x}_{2}, \mathrm{x}_{1}\right)+\mathrm{m}_{2}^{*} \mathrm{a}_{1}^{2} \Phi_{1}\left(\mathrm{x}_{2}, \mathrm{x}_{1}\right)+\mathrm{q}_{5}^{*} \frac{\lambda_{0} \mathrm{a}_{1}^{2}}{3} \Phi_{3}\left(\mathrm{x}_{2}, \mathrm{x}_{1}, \mathrm{a}_{1}\right)-\right. \\
& -\mathrm{q}_{4}^{*}\left[\frac{\mathrm{D}_{0}}{\mathrm{~A}_{1}}-\frac{\lambda_{0} \mathrm{a}_{1}^{2}}{42}\right] \Phi_{3}\left(\mathrm{x}_{2}, \mathrm{x}_{1}, \mathrm{a}_{1}\right)+\mathrm{m}_{1}^{*} \frac{\mathrm{D}_{0}}{\mathrm{~A}_{1}} \Phi_{5}\left(\mathrm{x}_{2}, \mathrm{x}_{1}, \mathrm{a}_{1}\right)+\mathrm{m}_{2}^{*} \frac{\mathrm{D}_{0}}{\mathrm{~A}_{1}} \Phi_{4}\left(\mathrm{x}_{2}, \mathrm{x}_{1}, \mathrm{a}_{1}\right)- \\
& -\mathrm{q}_{4}^{*} \mathrm{a}_{1}^{2} \Phi_{6}\left(\mathrm{x}_{2}, \mathrm{x}_{1}\right)-2 \mathrm{~m}_{1}^{*} \Phi_{5}\left(\mathrm{x}_{2}, \mathrm{x}_{1}, \mathrm{~b}_{1}\right)+2 \mathrm{~m}_{2}^{*} \Phi_{5}\left(\mathrm{x}_{1}, \mathrm{x}_{2}, \mathrm{~b}_{1}\right)+ \\
& +\mathrm{m}_{1}^{*} \mathrm{a}_{1}^{2} v \Phi_{1}\left(\mathrm{x}_{1}, \mathrm{x}_{2}\right)+\mathrm{m}_{2}^{*} \mathrm{a}_{1}^{2} v \Phi_{2}\left(\mathrm{x}_{1}, \mathrm{x}_{2}\right)+\mathrm{q}_{5}^{*} \frac{\lambda_{0} \mathrm{a}_{1}^{2} \mathrm{v}}{3} \Phi_{3}\left(\mathrm{x}_{1}, \mathrm{x}_{2}, \mathrm{a}_{1}\right)- \\
& -\mathrm{q}_{4}^{*} v\left[\frac{\mathrm{D}_{0}}{\mathrm{~A}_{1}}-\frac{\lambda_{0} \mathrm{a}_{1}^{2}}{42}\right] \Phi_{3}\left(\mathrm{x}_{1}, \mathrm{x}_{2}, \mathrm{a}_{1}\right)+\mathrm{m}_{1}^{*} \frac{v \mathrm{D}_{0}}{\mathrm{~A}_{1}} \Phi_{4}\left(\mathrm{x}_{1}, \mathrm{x}_{2}, \mathrm{a}_{1}\right)+\mathrm{m}_{2}^{*} \frac{v \mathrm{D}_{0}}{\mathrm{~A}_{1}} \Phi_{5}\left(\mathrm{x}_{1}, \mathrm{x}_{2}, \mathrm{a}_{1}\right)- \\
& \left.-q_{4}^{*} a_{1}^{2} v \Phi_{6}\left(x_{1}, x_{2}\right)\right\}+\frac{\lambda_{0}^{2} \Omega_{0}}{6 \pi A_{1}}\left\{q_{4}^{*}\left[1+\frac{A_{1} a_{1}^{2}}{14 \lambda_{0} \Omega_{0}}\right] \Phi_{7}\left(x_{1}, x_{2}\right)+q_{5}^{*} \frac{A_{1} a_{1}^{2}}{\lambda_{0} \Omega_{0}} \Phi_{7}\left(x_{1}, x_{2}\right)-\right. \\
& \left.-\mathrm{m}_{1}^{*} \Phi_{8}\left(\mathrm{x}_{1}, \mathrm{x}_{2}\right)-\mathrm{m}_{2}^{*} \Phi_{8}\left(\mathrm{x}_{2}, \mathrm{x}_{1}\right)\right\} \text {, } \\
& \mathrm{H}=-\frac{1-v}{2 \pi}\left\{\mathrm{m}_{1}^{*} \mathrm{a}_{1}^{2} \Phi_{2}\left(\mathrm{x}_{1}, \mathrm{x}_{2}\right)+\mathrm{m}_{2}^{*} \mathrm{a}_{1}^{2} \Phi_{2}\left(\mathrm{x}_{2}, \mathrm{x}_{1}\right)+\mathrm{q}_{5}^{*} \frac{\lambda_{0} \mathrm{a}_{1}^{2}}{3} \Phi_{9}\left(\mathrm{x}_{1}, \mathrm{x}_{2}, \mathrm{a}_{1}\right)-\right. \\
& -\mathrm{q}_{4}^{*}\left[\frac{\mathrm{D}_{0}}{\mathrm{~A}_{1}}-\frac{\lambda_{0} \mathrm{a}_{1}^{2}}{42}\right] \Phi_{9}\left(\mathrm{x}_{1}, \mathrm{x}_{2}, \mathrm{a}_{1}\right)+\mathrm{m}_{1}^{*} \frac{\mathrm{D}_{0}}{\mathrm{~A}_{1}} \Phi_{5}\left(\mathrm{x}_{1}, \mathrm{x}_{2}, \mathrm{a}_{1}\right)+\mathrm{m}_{2}^{*} \frac{\mathrm{D}_{0}}{\mathrm{~A}_{1}} \Phi_{5}\left(\mathrm{x}_{2}, \mathrm{x}_{1}, \mathrm{a}_{1}\right)- \\
& -\mathrm{q}_{4}^{*} \mathrm{a}_{1}^{2} \Phi_{10}\left(\mathrm{x}_{1}, \mathrm{x}_{2}\right)-\mathrm{m}_{1}^{*} \frac{1}{v-1} \Phi_{4}\left(\mathrm{x}_{2}, \mathrm{x}_{1}, \mathrm{~b}_{1}\right)-\mathrm{m}_{2}^{*} \frac{1}{v-1} \Phi_{4}\left(\mathrm{x}_{1}, \mathrm{x}_{2}, \mathrm{~b}_{1}\right)+ \\
& \left.+\mathrm{m}_{1}^{*} \frac{1}{v-1} \Phi_{5}\left(\mathrm{x}_{1}, \mathrm{x}_{2}, \mathrm{~b}_{1}\right)+\mathrm{m}_{2}^{*} \frac{1}{v-1} \Phi_{5}\left(\mathrm{x}_{2}, \mathrm{x}_{1}, \mathrm{~b}_{1}\right)\right\}, \\
& \mathrm{Q}_{10}=\frac{1}{2 \pi}\left\{\mathrm{m}_{1}^{*} \mathrm{~b}_{1}^{2} \Phi_{3}\left(\mathrm{x}_{2}, \mathrm{x}_{1}, \mathrm{~b}_{1}\right)-\mathrm{m}_{2}^{*} \mathrm{~b}_{1}^{2} \Phi_{9}\left(\mathrm{x}_{1}, \mathrm{x}_{2}, \mathrm{~b}_{1}\right)-\right. \\
& \left.-\mathrm{q}_{4}^{*} \Phi_{8}\left(\mathrm{x}_{1}, \mathrm{x}_{2}\right)-\mathrm{q}_{4}^{*} \mathrm{a}_{1}^{2} \Phi_{11}\left(\mathrm{x}_{1}, \mathrm{x}_{2}\right)\right\} \text {, } \\
& \mathrm{Q}_{20}=\frac{1}{2 \pi}\left\{-\mathrm{m}_{1}^{*} \mathrm{~b}_{1}^{2} \Phi_{9}\left(\mathrm{x}_{1}, \mathrm{x}_{2}, \mathrm{~b}_{1}\right)+\mathrm{m}_{2}^{*} \mathrm{~b}_{1}^{2} \Phi_{3}\left(\mathrm{x}_{1}, \mathrm{x}_{2}, \mathrm{~b}_{1}\right)-\right. \\
& \left.-\mathrm{q}_{4}^{*} \Phi_{8}\left(\mathrm{x}_{2}, \mathrm{x}_{1}\right)-\mathrm{q}_{4}^{*} \mathrm{a}_{1}^{2} \Phi_{11}\left(\mathrm{x}_{2}, \mathrm{x}_{1}\right)\right\} \text {, }
\end{aligned}
$$




$$
\begin{aligned}
& \mathrm{Q}_{12}=\frac{1}{28 \pi}\left\{-\mathrm{m}_{1}^{*} \mathrm{~b}_{1}^{2} \Phi_{3}\left(\mathrm{x}_{2}, \mathrm{x}_{1}, \mathrm{~b}_{1}\right)+\mathrm{m}_{2}^{*} \mathrm{~b}_{1}^{2} \Phi_{9}\left(\mathrm{x}_{1}, \mathrm{x}_{2}, \mathrm{~b}_{1}\right)+\mathrm{m}_{1}^{*} \frac{14 \lambda_{0} \Omega_{0}}{\mathrm{~A}_{1}} \Phi_{3}\left(\mathrm{x}_{1}, \mathrm{x}_{2}, \mathrm{a}_{1}\right)+\right. \\
& \left.+\mathrm{m}_{2}^{*} \frac{14 \lambda_{0} \Omega_{0}}{\mathrm{~A}_{1}} \Phi_{9}\left(\mathrm{x}_{1}, \mathrm{x}_{2}, \mathrm{a}_{1}\right)-14 \mathrm{q}_{5}^{*} \Phi_{8}\left(\mathrm{x}_{1}, \mathrm{x}_{2}\right)+\mathrm{q}_{4}^{*}\left[\mathrm{a}_{1}^{2}+\frac{14 \lambda_{0} \Omega_{0}}{\mathrm{~A}_{1}}\right] \Phi_{11}\left(\mathrm{x}_{1}, \mathrm{x}_{2}\right)\right\}, \\
& \mathrm{Q}_{22}=\frac{1}{28 \pi}\left\{\mathrm{m}_{1}^{*} \mathrm{~b}_{1}^{2} \Phi_{9}\left(\mathrm{x}_{1}, \mathrm{x}_{2}, \mathrm{~b}_{1}\right)-\mathrm{m}_{2}^{*} \mathrm{~b}_{1}^{2} \Phi_{3}\left(\mathrm{x}_{1}, \mathrm{x}_{2}, \mathrm{~b}_{1}\right)+\mathrm{m}_{1}^{*} \frac{14 \lambda_{0} \Omega_{0}}{\mathrm{~A}_{1}} \Phi_{9}\left(\mathrm{x}_{1}, \mathrm{x}_{2}, \mathrm{a}_{1}\right)+\right. \\
& \left.+\mathrm{m}_{2}^{*} \frac{14 \lambda_{0} \Omega_{0}}{\mathrm{~A}_{1}} \Phi_{3}\left(\mathrm{x}_{2}, \mathrm{x}_{1}, \mathrm{a}_{1}\right)-14 \mathrm{q}_{5}^{*} \Phi_{8}\left(\mathrm{x}_{2}, \mathrm{x}_{1}\right)+\mathrm{q}_{4}^{*}\left[\mathrm{a}_{1}^{2}+\frac{14 \lambda_{0} \Omega_{0}}{\mathrm{~A}_{1}}\right] \Phi_{11}\left(\mathrm{x}_{2}, \mathrm{x}_{1}\right)\right\}, \\
& \mathrm{R}_{1}=\frac{\lambda_{0} \Omega_{0}}{6 \pi \mathrm{A}_{1}}\left\{\mathrm{q}_{4}^{*}\left[1+\frac{\mathrm{A}_{1} \mathrm{a}_{1}^{2}}{14 \lambda \lambda_{0} \Omega_{0}}\right] \Phi_{7}\left(\mathrm{x}_{1}, \mathrm{x}_{2}\right)+\mathrm{q}_{5}^{*} \frac{\mathrm{A}_{1} \mathrm{a}_{1}^{2}}{\lambda_{0} \Omega_{0}} \Phi_{7}\left(\mathrm{x}_{1}, \mathrm{x}_{2}\right)-\right. \\
& \left.-\mathrm{m}_{1}^{*} \Phi_{8}\left(\mathrm{x}_{1}, \mathrm{x}_{2}\right)-\mathrm{m}_{2}^{*} \Phi_{8}\left(\mathrm{x}_{2}, \mathrm{x}_{1}\right)\right\},
\end{aligned}
$$

where

$$
\begin{gathered}
\Phi_{1}\left(x_{1}, x_{2}\right)=-\frac{3}{8} x_{1} G_{1,0}\left(a_{1} \sqrt{x_{1}^{2}+x_{2}^{2}}\right)-\frac{x_{1}\left(x_{1}^{2}-3 x_{2}^{2}\right)}{8\left(x_{1}^{2}+x_{2}^{2}\right)} G_{2,1}\left(a_{1} \sqrt{x_{1}^{2}+x_{2}^{2}}\right), \\
\Phi_{2}\left(x_{1}, x_{2}\right)=-\frac{1}{8} x_{2} G_{1,0}\left(a_{1} \sqrt{x_{1}^{2}+x_{2}^{2}}\right)-\frac{x_{2}\left(3 x_{1}^{2}-x_{2}^{2}\right)}{8\left(x_{1}^{2}+x_{2}^{2}\right)} G_{2,1}\left(a_{1} \sqrt{x_{1}^{2}+x_{2}^{2}}\right), \\
\Phi_{3}\left(x_{1}, x_{2}, c\right)=\frac{1}{2}\left[G_{0,0}\left(c \sqrt{x_{1}^{2}+x_{2}^{2}}\right)+\frac{x_{1}^{2}-x_{2}^{2}}{x_{1}^{2}+x_{2}^{2}} G_{1,1}\left(c \sqrt{x_{1}^{2}+x_{2}^{2}}\right)\right], \\
\Phi_{4}\left(x_{1}, x_{2}, c\right)=\frac{3 x_{1}}{2\left(x_{1}^{2}+x_{2}^{2}\right)} G_{0,1}\left(c \sqrt{x_{1}^{2}+x_{2}^{2}}\right)+\frac{x_{1}\left(x_{1}^{2}-3 x_{2}^{2}\right)}{2\left(x_{1}^{2}+x_{2}^{2}\right)^{2}} G_{1,2}\left(c \sqrt{x_{1}^{2}+x_{2}^{2}}\right), \\
\Phi_{5}\left(x_{1}, x_{2}, c\right)=\frac{x_{2}}{2\left(x_{1}^{2}+x_{2}^{2}\right)} G_{0,1}\left(c \sqrt{x_{1}^{2}+x_{2}^{2}}\right)+\frac{x_{2}\left(3 x_{1}^{2}-x_{2}^{2}\right)}{2\left(x_{1}^{2}+x_{2}^{2}\right)^{2}} G_{1,2}\left(c \sqrt{x_{1}^{2}+x_{2}^{2}}\right), \\
\Phi_{6}\left(x_{1}, x_{2}\right)=-\frac{1}{2 a_{1}^{2}} \ln \frac{\gamma \sqrt{x_{1}^{2}+x_{2}^{2}}}{2}-\frac{1}{2 a_{1}^{2}} G_{0,0}\left(a_{1} \sqrt{x_{1}^{2}+x_{2}^{2}}\right)-\frac{x_{1}^{2}-x_{2}^{2}}{8} G_{2,0}\left(a_{1} \sqrt{x_{1}^{2}+x_{2}^{2}}\right), \\
\Phi_{7}\left(x_{1}, x_{2}\right)=G_{0,0}\left(a_{1} \sqrt{x_{1}^{2}+x_{2}^{2}}\right), \Phi_{8}\left(x_{1}, x_{2}\right)=\frac{2 x_{1}}{x_{1}^{2}+x_{2}^{2}} G_{0,1}\left(a_{1} \sqrt{x_{1}^{2}+x_{2}^{2}}\right), \\
x_{1}^{2} x_{2} G_{1,1}\left(c \sqrt{x_{1}^{2}+x_{2}^{2}}\right), \Phi_{10}\left(x_{1}, x_{2}\right)=-\frac{x_{1} x_{2}}{4} G_{2,0}\left(a_{1} \sqrt{x_{1}^{2}+x_{2}^{2}}\right), \\
\left.a_{1} \sqrt{x_{1}^{2}+x_{2}^{2}}\right),
\end{gathered}
$$

where $\mathrm{G}_{\mathrm{n}, \mathrm{v}}(\mathrm{rz})$ - special G-function [12].

When the cyclic change of variables in (6) $\left(\mathrm{x}_{1}\right.$ on $\mathrm{x}_{2}$ and $\mathrm{x}_{2}$ on $\left.\mathrm{x}_{1}\right)$, we obtain relevant $\Phi_{\mathrm{i}}\left(\mathrm{x}_{2}, \mathrm{x}_{1}\right)(\mathrm{i}=\overline{1,10})$.

4. Investigation results for behavior of the stress-strain state components depending on the elastic constants of transversely isotropic material and their discussion

To investigation of the effect of the elastic constants on the stress-strain state components of transversely isotropic plates under concentrated force effects we set $\mathrm{m}_{1}^{*}=\mathrm{m}_{2}^{*}=\mathrm{q}_{4}^{*}=\mathrm{q}_{5}^{*}=1$. 
The results of calculations are presented in the dimensionless Cartesian coordinate system $\mathrm{x}_{1}, \mathrm{x}_{2}$. Graphs are built along the $\mathrm{x}$-axis $\left(\mathrm{x}_{2}=0\right)$.

Numerical calculations are performed for two kinds of plate material: isotropic $\left(\mathrm{E}^{*}=1\right.$; $\left.\mathrm{E} / \mathrm{G}^{\prime}=2,6 ; v=v^{\prime}=0,3\right)$ and transversely isotropic $\left(\mathrm{E}^{*}=5 ; \mathrm{v}=0,3 ; v^{\prime}=0,07\right)$. Values for the shear compliance of this material $\mathrm{E} / \mathrm{G}^{\prime}$ are consistent with such values: 40, 80, 120. These values are given for a transversely isotropic material in the paper [3].

Fig. 1-3 demonstrate dependence of $\mathrm{Q}_{10}, \mathrm{Q}_{12}, \mathrm{R}_{1}$ on the parameter of the shear compliance $\mathrm{E} / \mathrm{G}^{\prime}$. The green curve corresponds to a value of 2.6, and the red, blue and black curves - values of 40, 80 and 120 , respectively.

On these graphs (Fig. 1-3) it can be seen that with increasing shear compliance parameter $\mathrm{E} / \mathrm{G}^{\prime}$, considered $\mathrm{Q}_{10}, \mathrm{Q}_{12}, \mathrm{R}_{1}$ are increasing in magnitude.

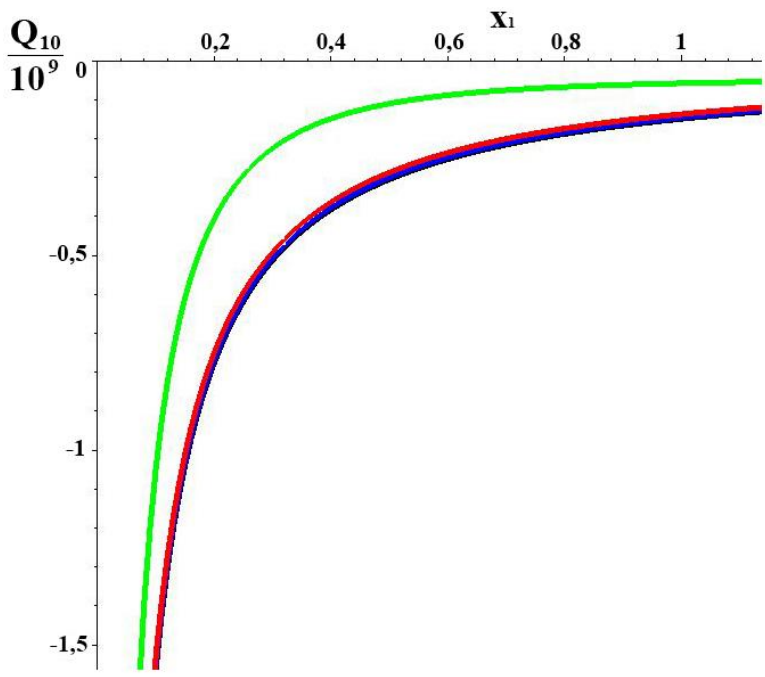

Fig. 1. The shear force $\mathrm{Q}_{10}$ : green curve - the parameter of shear compliance corresponds to a value of 2.6; red, blue and black curves - the values of 40,80 and 120, respectively; $\mathrm{x}_{1}$ - the $\mathrm{x}$ axis

$$
\left(\mathrm { x } _ { 1 } = \mathrm { x } / \mathrm { h } \text { , where } \mathrm { h } - \text { the half thickness of the plate) } ; \frac { \mathrm { Q } _ { 1 0 } } { 1 0 ^ { 9 } } - \mathrm { Y } \text { -axis } \left(\mathrm{Q}_{10}=\frac{\mathrm{Q}_{\mathrm{x} 0}}{\text { Eh }},\right.\right.
$$

where E - Young's modulus, $\mathrm{h}$ - the half thickness of the plate, $10^{9}$ - the scaling factor)

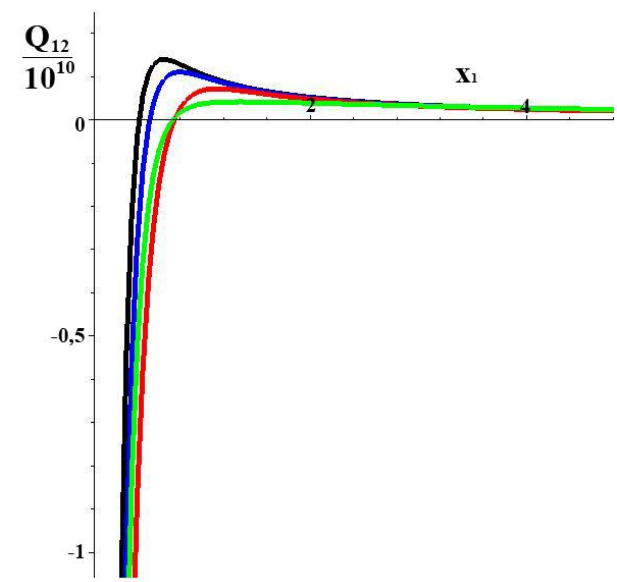

Fig. 2. The shear force $\mathrm{Q}_{12}$ : green curve - the parameter of shear compliance corresponds to a value of 2.6; red, blue and black curves - the values of 40,80 and 120, respectively; $\mathrm{x}_{1}$ - the $\mathrm{x}$ axis

$$
\left(\mathrm { x } _ { 1 } = \mathrm { x } / \mathrm { h } \text { , where } \mathrm { h } - \text { the half thickness of the plate); } \frac { \mathrm { Q } _ { 1 0 } } { 1 0 ^ { 9 } } - \mathrm { Y } \text { -axis } \left(\mathrm{Q}_{10}=\frac{\mathrm{Q}_{\mathrm{x} 0}}{\mathrm{Eh}},\right.\right.
$$

where $\mathrm{E}$ - Young's modulus, $\mathrm{h}$ - the half thickness of the plate, $10^{9}$ - the scaling factor) 


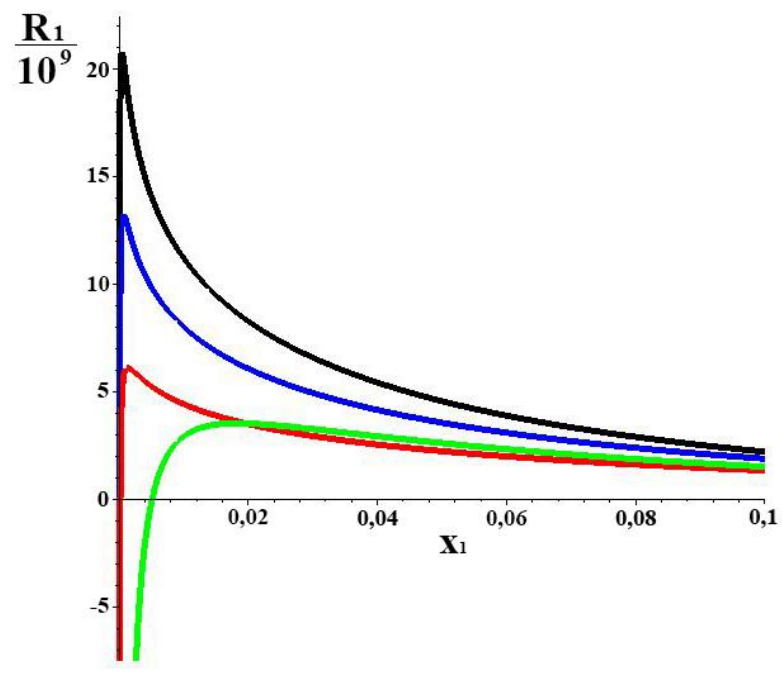

Fig. 3. The generalized force $\mathrm{R}_{1}$ : green curve - the parameter of shear compliance corresponds to a value of 2.6; red, blue and black curves - the values of 40, 80 and 120, respectively; $\mathrm{x}_{1}-$ the $\mathrm{x}$ axis $\left(\mathrm{x}_{1}=\mathrm{x} / \mathrm{h}\right.$, where $\mathrm{h}$ - the half thickness of the plate $) ; \frac{\mathrm{R}_{1}}{10^{9}}-\mathrm{Y}$-axis $\left(\mathrm{R}_{1}=\frac{\mathrm{R}_{1}}{\mathrm{Eh}}\right.$, where E - Young's modulus, $\mathrm{h}$ - the half thickness of the plate, $10^{9}$ - the scaling factor)

Estimates allow investigating the behavior of the generalized forces, depending on the value of the shear compliance.

\section{Conclusions}

Thus, our studies suggest that in the calculation of thin-walled elements of constructions made of advanced composite materials on concentrated forces it is necessary to use the refined theory of plates and shells. These theories allow estimating the phenomena associated with taking into account the transverse shear and compression.

The practical significance of the results is the ability to use them in calculations related to the design and definition of the operating parameters of thin-walled elements of constructions containing the stress concentrator. The results can be used in scientific research institutes, design organizations and other research institutions involved in the calculations of thin-walled elements of constructions.

\section{References}

[1] Pogorelov, V. I. (2007). Structural Mechanics of thin-walled structures: a tutorial. Saint Peterburg: BHV-Petersburg, 518.

[2] Bashev, V. F., Sukhov, E. V., Syrovatko, J. V. (2012). Statistical analysis of microstructure of composite materials. Starodubovskie reading, 53-57.

[3] Pelekh, B. L., Lazko, V. A. (1982). Laminated anisotropic plates and shells with stress concentrators. Kyiv: Science. Dumka, 296.

[4] Bokov, I. P., Strelnikova, E. A. (2015). Fundamental solution of static equations of transversely isotropic plates. International Journal of Innovative Research in Engineering and Management, 2 (6), 56-62.

[5] Bokov, I. P., Bondarenko, N. S., Strelnikova, E. A. (2016). Construction of the fundamental solution of the equations of statics $\{1,2\}$-approximation the membrane stress state for transversely isotropic plates. ScienceRise, 8 (2 (25)), 41-48. doi: 10.15587/2313-8416.2016.76534 
[6] Bondarenko, N. S., Goltsev, A. S. (2015). Research incision influence the stress intensity factors in an isotropic plate on the basis of the generalized theory. Proceedings of the Institute of Applied. Mathematics and Mechanics, 29, 20-28.

[7] Bondarenko, N. S., Goltsev, A. S. (2010). Fundamental solutions of the equations of thermoelasticity transversely isotropic plates. Journal of Applied Mechanics, 46 (3), 51-60.

[8] Bondarenko, N. S. (2009). The fundamental solution of differential equations of thermoelasticity $\{1,0\}$-approximation for transversely isotropic plates. Proceedings of the Institute of Applied Mathematics and Mechanics of National Academy of Sciences of Ukraine, 18, 11-18.

[9] Bondarenko, N. S., Goltsev, A. S., Shevchenko, V. P. (2009). Fundamental solution $\{1,2\}$-approximation the membrane thermoelastic state transversely isotropic plates. Reports of National Academy of Sciences of Ukraine, 11, 46-52.

[10] Vladimirov, V. S. (1976). Generalized functions in mathematical physics. Moscow: Science, 280.

[11] Sneddon, I. (1955). Fourier transform. Moscow: Foreign literature publishing house, 668.

[12] Khizhnyak, V. K., Shevchenko, V. P. (1980). Mixed problem in the theory of plates and shells: a tutorial. Donetsk: DonGU, 128. 